\title{
High flow nasal cannula in patients treated inside and outside the pediatric intensive care units: pending challenges
}

\begin{abstract}
High Flow Nasal Cannula (HFNC) is a non-invasive oxygen therapy system it consists in the administration of gas flows, mixture of air and oxygen $\left(\mathrm{O}_{2}\right)$, which exceed the patient's ventilatory demands and also it is a friendly system, which allows its use outside of critical units.

Objective: To collect and analyse the available literature in relation to the definition and clinical parameters that predict the failure of HFNC therapy, in hospitalized pediatric patients inside and outside the ICU, in order to provide knowledge to clinicians to optimize the management of this device, facilitate the administration of the resources and finally, improve the pediatric patients outcomes by identifying the clinical predictors of HFNC failure.

Conclusion: There is no consensus regarding the definition of failure of HFNC, which makes it difficult to interpretate the results of the studies and, therefore, its translation into clinical practice. On the other hand, although predictive factors of failure have been identified, in most studies, are restricted to demographic characteristics, comorbidities, severity indexes and/or require the taking of arterial blood tests, therefore their applicability outside of the Pediatric Intensive Care Unit (PICU), is limited. Regarding the identification of clinical parameters, some of the studies mentioned their correlation with failure, but during the administration of the therapy and not previously. This situation makes the early characterization of patients who require HFNC more difficult, and therefore, the individualized decisions that could eventually improve economical and clinical outcomes of our pediatric patients.
\end{abstract}

Keywords: high flow nasal cannula, nasal high flow, oxygen therapy, paediatrics [mesh], predictors, failure
Volume 4 Issue 2 - 2019

\section{Betel Rivero-Campos, Ana Urzúa, Patricia Vargas, Juan Eduardo Keymer,Viviane Hidalgo-Cabalín, Tania Gutiérrez-Panchana \\ Physical Medicine and Rehabilitation Service, Clínica Alemana, Chile}

Correspondence: Betel Rivero-Campos, Physical Medicine and Rehabilitation Service, Clínica Alemana, Santiago,Vitacura, Santiago, Chile, Tel +56995980349, Email briveroc@alemana.cl. klga.rivero@gmail.com

Received: April 05, 2019 | Published: April 17, 2019
Abbreviations: HFNC, high flow nasal cannula; NIV, noninvasive ventilation; MV, mechanical ventilation; PRISM-III pediatric risk of mortality III; PICU, pediatric intensive care unit

\section{Introduction}

High Flow Nasal Cannula (HFNC) is a non-invasive oxygen therapy system, initially used in the neonatal population and currently widely used in pediatric and adult patients. ${ }^{1}$ It consists in the administration of gas flows, mixture of air and oxygen $\left(\mathrm{O}_{2}\right)$, which exceed the patient's ventilatory demands. ${ }^{2}$ Its main mechanisms of action are the increase of inspired fraction of oxygen $\left(\mathrm{F}_{\mathrm{i}} \mathrm{O}_{2}\right)$ washing of the nasopharyngeal dead space, increase of the final expiratory volume and functional residual capacity, provision of correct humidification to the airway and decrease of inspiratory resistance. ${ }^{3,4}$

Along with Non-Invasive Ventilation (NIV), HFNC seek to treat respiratory failure avoiding the need for invasive Mechanical Ventilation (MV), and preventing post-extubation failure. At the same time HFNC has multiple advantages over other supports, for example: (1) it favours the gas mixture to be heated and humidified to reduce the risk of damage of the upper airway mucous, (2) the concentration of $\mathrm{O}_{2}$ can be precisely titled, (3) it favours better tolerance and (4) helps to reduce work of breath and the need for greater ventilatory support. ${ }^{5}$ Furthermore, it is a friendly system, which allows its use outside of critical units. ${ }^{6,7}$
Abboud et al. $^{8}$ defines failure of HFNC as the intubation requirement. In this study the average weight and the weight corrected by age were significantly lower in the group of patients who required intubation ( $p=0.016$ and $p=0.031$, respectively). On the other hand, the Respiratory Rate (RR), at the beginning of the connection to HFNC, also correlates strongly with respiratory deterioration $(p<0.001)$ and carbon dioxide blood pressure was significantly higher before $(p<0.001)$ and after $(p<0.001)$ the therapy in non-responder group. Finally, the scores of Pediatric Risk of Mortality III (PRISMIII) in patients who failed, were significantly higher $(p<0.001)$, in comparison to the group of patients who presented good response to the therapy. ${ }^{8}$

The publication made by Kamit Can et al. ${ }^{9}$ in 2017, also studies children hospitalized in Pediatric Intensive Care Unit (PICU), particularly to the usefulness of the relationship between oxygen saturation and $\mathrm{F}_{\mathrm{i}} \mathrm{O}_{2}\left(\mathrm{SpO}_{2} / \mathrm{F}_{\mathrm{i}} \mathrm{O}_{2}\right)$, as an outcome predictor of HFNC therapy. This article included patients with distress or acute respiratory failure from one month to 18 years admitted to the PICU and treated with HFNC as primary support or as support after extubation. Failure of HFNC was defined as the need to scale up to NIV or MV. The responders and non-responders of HFNC were compared with the clinical data just before HFNC, at 30,60 and 120 minutes and at 12, 24 and 48 hours, as well as once the therapy was completed. Ages higher than 120 months, higher PRISM-III index and respiratory scores, and lower $\mathrm{SpO}_{2} / \mathrm{FiO}_{2}$ ratio at the time of admission were 
predictors of failure of HFNC. Reach of a $\mathrm{SpO}_{2} / \mathrm{FiO}_{2}$ ratio higher than 200 at 60 minutes significantly predicted the success of the HFNC. ${ }^{9}$

Betters et al, refer to pediatric population hospitalized outside the PICU. In his study, the objective was to characterize patient qualities associated with failure of HFNC. Failure was defined as the requirement for intubation or cardiopulmonary arrest event. Twosample tests and binary logistic regression revealed that patients were more likely to suffer failure of HFNC if they had a history of cardiac disease $(\mathrm{p}=0.026)$, intubation history $(\mathrm{p}=0.040)$ and required a higher $\mathrm{F}_{\mathrm{i}} \mathrm{O}_{2}(\mathrm{p}=<0.001) .{ }^{10}$

Predictors of failure in pediatric patients connected to HFNC in Emergency Services have also been analysed. Hansen et al reported that non-responders to HFNC are those who required admission to the PICU. Continuous clinical evaluation of the patient is proposed by using a Pediatric Early Warning Score (PEWS). Non-responders had higher PEWS scores at 90 minutes after the beginning of HFNC. Eighty percent of non-responders required NIV. In both groups, responders and non-responders, there were no requirements for intubation, air leaks or difference in days of ventilatory support. ${ }^{11}$

The objective of this study is to collect and analyse the available literature in relation to the definition and clinical parameters that predict the failure of HFNC therapy, in hospitalized pediatric patients inside and outside the ICU, in order to provide knowledge to clinicians to optimize the management of this device, favour the administration of the resources and finally, improve the pediatric patients outcomes by identifying the clinical predictors of HFNC failure.

\section{Discussion}

Despite the multiple studies available and the advance in the knowledge of the management of HFNC, in pediatric patients, it is still pending to agree on a single definition of failure, in order to establish criteria and use a common language between clinicians. This does not allow us to identify early clinical criteria of failure, and eventually, recognize patients who are in a more fragile situation to give them adequate ventilatory support attending their needs in a timely manner.

Although, our results help us to develop a profile of the patient that is susceptible to failure during the use of the HFNC, they have limited application in patients hospitalized outside the PICU, where the sampling of arterial blood gases, is not considered a routine practice and does not apply the calculation with PRISM-III. On the other hand, our population of interest covers a wide variety of diagnoses by which a child might need HFNC, as bronchiolitis, pneumonia or asthma. Finally, many studies limit themselves to associate demographic characteristics or comorbidities with the failure of HFNC, instead of relating hemodynamic and respiratory parameters previous or during the administration of therapy.

Another pending aspect to explore in pediatric patients is the incorporation of combined indexes, such as the ROX index, which considers the relationship between $\mathrm{SpO}_{2} / \mathrm{F}_{\mathrm{i}} \mathrm{O}_{2}$ and RR. Roca et al, evaluates its predictive value to decide intubation in adult patients. A ROX index higher than 4.88, measured at 2, 6 and 12 hours after the beginning of HFNC was consistently associated with a lower risk of intubation. By contrary, a ROX index lower than 2.85 at 2 hours, 3.47 at 6 hours and 3.85 at 12 hours, were predictors of failure. ${ }^{12}$

\section{Conclusion}

In conclusion, there is no consensus regarding the definition of failure of HFNC, which makes it difficult to interpretate the results of the studies and, therefore, its translation into clinical practice. On the other hand, although predictive factors of failure have been identified, in most studies, are restricted to demographic characteristics, comorbidities, severity indexes and/or require the taking of arterial blood tests, therefore their applicability outside of the PICU, is limited. Regarding the identification of clinical parameters, some of the studies mentioned their correlation with failure, but during the administration of the therapy and not previously. This situation makes the early characterization of patients who require HFNC more difficult, and therefore, the individualized decisions that could eventually improve economical and clinical outcomes of our pediatric patients.

\section{Acknowledgments}

None

\section{Conflicts of interest}

The authors declare no conflicts of interest.

\section{References}

1. Mikalsen, Peter D, Knut Ø. High flow nasal cannula in children: a literature review. Scandinavian Journal of Trauma, Resuscitation and Emergency Medicine. 2016;24:93.

2. Milési C, Essouri S, Pouyau R, et al. High flow nasal cannula (HFNC) versus nasal continuous positive airway pressure (nCPAP) for the initial respiratory management of acute viral bronchiolitis in young infants: a multicenter randomized controlled trial (TRAMONTANE study). Intensive Care Med. 2017; 43(2):209-216.

3. Frizzola M, Miller TL, Rodriguez ME, et al. High-flow nasal cannula: impact on oxygenation and ventilation in an acute lung injury model. Pediatr Pulmonol. 2011;46(1):67-74.

4. Dysart K, Miller TL, Wolfson MR, et al. Research in high flow therapy: mechanisms of action. Respir Med. 2009;103(10):1400-1405.

5. Cuquemelle E, Pham T, Papon JF, et al. Heated and humidified highflow oxygen therapy reduces discomfort during hypoxemic respiratory failure. Respir Care. 2012;57(10):1571-1577.

6. Rubin S, Ghuman A, Deakers T, et al. Effort of breathing in children receiving high-flow nasal cannula. Pediatr Crit Care Med. 2014;15(1):16.

7. Parke RL, McGuinness SP, Eccleston ML. A preliminary randomized controlled trial to assess effectiveness of nasal high-flow oxygen in intensive care patients. Respir Care. 2011;56(3):265-270.

8. Abboud PA, Roth PJ, Skiles CL, et al. Predictors of failure in infants with viral bronchiolitis treated with high-flow, high-humidity nasal cannula therapy. Pediatr Crit Care Med. 2012;13(6):e343-349.

9. Kamit CF, Anil AB, Anil M, et al. Predictive factors for the outcome of high flow nasal cannula therapy in a pediatric intensive care unit: Is the SpO2/FiO2 ratio useful?. J Crit Care. 2018;44:436-444.

10. Betters KA, Gillespie SE, Miller J, et al. High flow nasal cannula use outside of the ICU; factors associated with failure. Pediatr Pulmonol. 2017;52(6):806-812.

11. Hansen G, Hochman J, Garner M, et al. Pediatric early warning scores and declining ward patients on high flow therapy. Pediatr Int. 2019;61(3):278-283.

12. Roca $\mathrm{O}$, Caralt $\mathrm{B}$, Messika J, et al. An index combining respiratory rate and oxygenation to predict outcome of nasal high flow therapy. Am J Respir Crit Care Med. 2018:21. 